\title{
Effects of Acacia nilotica Leaf Extract on Adrenaline-induced Hyperlipidemia and Cardiac Remodeling in Rats
}

\author{
Nazmun Naher ${ }^{1}$, A.H.M. Khurshid Alam¹, Saiful Islam¹, Al-Mamun², AKM Motahar Hossain', \\ Md. Aziz Abdur Rahman ${ }^{1}$ and Mamunur Rashid ${ }^{1}$ \\ ${ }^{1}$ Department of Pharmacy, University of Rajshahi, Rajshahi, Bangladesh \\ ${ }^{2}$ Department of Pharmacy, Jessore Science and Technology University, Jessore, Bangladesh
}

\begin{abstract}
Hyperlipidemia is one of the major causes of disability in western as well as in developing countries. The present study was designed to investigate the effect of the ethanolic leaf extract of a Bangladeshi medicinal plant Acacia nilotica (AN) on adrenaline-induced hyperlipidemia and cardiac remodeling in rats. The plant extract and commercial lipid lowering drug atorvastatine were administered in adrenaline-induced hyperlipidemic rats (AIHRs) through intraperitoneal route for one week at a dose of $30 \mathrm{mg}$ and $0.04 \mathrm{mg}$ per $150 \mathrm{gm}$ body weight of rat, respectively. The AN extract showed a significant $(P<0.01)$ decrease in lipid profile, and reducing the left ventricular hypertrophy (heart weight/body weight), heart weight, cardiac myocytes size as well as kidney, pancreas and liver weight of AIHRs. The observation was also made for consecutive four weeks (long term treatment) to confirm the results obtained in one week model. The results indicated that the leaf extract of A. nilotica in comparison with atorvastatine has got profound hypolipidemic and anticardiac remodeling activities.
\end{abstract}

Key words: Hyperlipidemia, Fabaceae, Acacia nilotica, Atorvastatine, Cardiac remodeling.

\section{Introduction}

Atherosclerosis and coronary heart disease are the major health problem in developed and modern societies and leading causes of death in diabetic patients (Stamler $e t$ al., 1993; Wilson, 2001). Coronary vessel disease and atherosclerosis being primary reasons for the increased incidence of cardiovascular dysfunction (Lteif et al., 2003). The American heart association estimates that $65 \%$ of patients with diabetes die due to some form of cardiovascular disease (CVD), hence, good control of blood sugar levels can reduce the risk of CVD (Bhopal and Rafnsson, 2007; Richard and Mary, 2007). Cardiac remodeling, defined as the alteration in the dimensions, mass, shape and function of the heart, is generally accepted as a determinant of the clinical course of heart failure (HF). Although patients with major remodeling demonstrate progressive worsening of cardiac function, slowing or reversing remodeling has only recently become a goal of HF therapy (Opie et al., 2006).

The use of traditional medicine has played an important role in nearly every culture on earth, including Asia, Africa, Europe and America. Traditional medicine is based on plants contain chemicals that can promote health and alleviate illness (Grabely and Thiericke, 1999). Herbal treatments are the most popular form of traditional medicine, and are highly lucrative in the international marketplace due to availability, low cost and less side effect. Some traditional medicinal plants have already been reported to work against cardiovascular diseases (Wong, 2011) as well as high blood cholesterol (Aattar, 2006).

Acacia nilotica, locally known as babla, belongs to the family fabaceae. The plant is widely distributed in the Indo-Bangla subcontinent and cultivated throughout the tropical belt. Different parts of this plant are reported to be used for the treatment of human aliments. The whole plant has antiplatelet aggregatory activity (Shah et al., 1995). Methanol extract of leaf of A. nilotica possesses hypotensive and hypospasmodic activities (Gilani et al., 1999). The leaves are also reported to be a good hypocholesterolemic agent (Ghasi et al., 2000). An aqueous extract of the seed of A. nilotica was shown to possess sustained dose-related contractile activity on isolated guinea-pig ileum (Amos et al., 1999). On the basis of the reported pharmacological activity, the plant was selected for hypolipidemic and anticardiac

Correspondence to: Mamunur Rashid, E-mail: mamun69jp@yahoo.com 
remodeling investigation and the aim of our present study is to investigate the effects of A. nilotica leaf extract on adrenaline induced hyperlipidemia and cardiac remodeling in rats.

\section{Materials and Methods}

Plant materials: Fresh leaves of the plant A. nilotica were collected from the Botanical Garden of the University of Rajshahi and authenticated at the Department of Botany, University of Rajshahi, Rajshahi, Bangladesh.

Preparation of crude leaf extract: The leaves of AN were dried completely under the mild sun and ground with an electric grinder into coarse powder. The powdered material was then extracted with $95 \%$ ethanol and room temperature for 7 days with occasional shaking and stirring. The extract was filtered through cotton filter and the filtrate was then concentrated with a rotary evaporator under reduced temperature and pressure to get brownish mass.

Animal studies: All protocols for the animal experiments were reviewed and approved by the animal care and use committee of Institute of Biological Science, University of Rajshahi. Long-Evans male rats weighing about 110-130 mg, aged 2 months, were purchased from animal's house of International Centre for Diarrhoeal Disease Research, Bangladesh (ICDDR,B). Prior to commencement of the experiments, all the rats were acclimatized to the new environmental condition for a period of one week. During the experimental period, the rats were kept in a well ventilated animal house at room temperature and were supplied standard pellets from ICDDRB and fresh drinking water. All the rats were kept in cages and maintained with natural 12 hour light and dark cycle. Adrenaline was injected intra-peritonially (i.p.) in rats. AIHRs were treated with i.p. injection of AN at various doses for dose selection. After completing the dose selection, AIHRs were treated with the most effective observed dose of AN (30 mg /150 gm b.w.) and adrenaline (0.04 mg/150 gm BW) for both 1 and 4 weeks.

Measurement of lipid profile: After completing the treatment with ethanolic extract of AN leaves and atorvastatin, the rats were first anesthetized with sodium phenobarbital. Then abdominal skin was cut, and thoracic artery was opened. Finally, 3-5 ml of blood was collected directly from the thoracic artery by syringes. At last, the blood was centrifuged at $4000 \mathrm{rpm}$ for 10 minutes, and the serum was obtained. Serum lipid profiles, such as TC, TG, LDL-C and HDL-C, were assessed using diagnostic kits (Human, Germany).

Histopathological analysis: Histological studies were performed for the determination of LV weight and cardiomyocyte cross sectional area. In brief, LV weight was measured, and the ratio of LV weight to body weight was calculated to determine an index of LV hypertrophy. Four sections were obtained from each heart, and mounted on slides and stained with hematoxylin and eosin. To evaluate the extent of cardiomyocyte hypertrophy, crosssectional images of cardiomyocyte were scanned at $\times 400$ magnifications. Approximately 20 cross-sections of cardiomyocyte were analyzed in each heart. Average values for each heart were used for analysis. All images were taken using an Olympus BX51TF (Olympus Corporation, Tokyo, Japan), and all measurements were determined using Scion Image software (Scion Corporation, Frederick, MD).

Statistical analysis: The results were expressed as mean \pm SEM. We used a one-way analysis of variance (ANOVA), followed by Dunnett's post-hoc test or students paired or unpaired $t$-test where appropriate. The statistical method applied in each analysis was described in each figure. Results were considered to be significant when $\mathrm{p}$ values were less than $0.05(\mathrm{p}<0.05)$.

\section{Results and Discussion}

Effects of AN leaf extract on TC, TG, LDLcholesterol, and HDL-cholesterol level in adrenaline induced hyperlipidemic rats (AIHRs): AIHRs were treated with ethanolic extract of AN at $30 \mathrm{mg} / 150 \mathrm{gm}$ b.w. for one week and it was observed that AN leaf extract significantly reduced total cholesterol level $(1.805 \mathrm{mmol} / \mathrm{l})$ compared to atorvastatine $(1.34 \mathrm{mmol} / \mathrm{l})$. AN leaf extract also reduced triglyceride level $(2.022 \mathrm{mmol} / \mathrm{l})$ as well as LDL-cholesterol level $(1.95 \mathrm{mmol} / \mathrm{l})$ and raises HDLcholesterol level $(1.50 \mathrm{mmol} / \mathrm{l})$ in adrenaline induced hyperlipidemic rats (Figure 1A-1D).

Four weeks treatment with AN extract also significantly reduced total cholesterol level $(1.34 \mathrm{mmol} / \mathrm{l})$, triglyceride level $(1.98 \mathrm{mmol} / \mathrm{l})$, and LDL-cholesterol level $(1.99 \mathrm{mmol} / \mathrm{l})$ and raised HDL-cholesterol level $(1.48 \mathrm{mmol} / \mathrm{l})$ in adrenaline induced hyperlipidemic rats (Figure 2A-2D). 


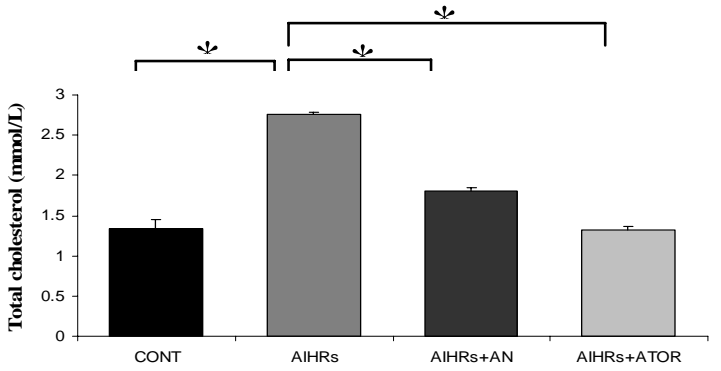

$1 \mathrm{~A}$

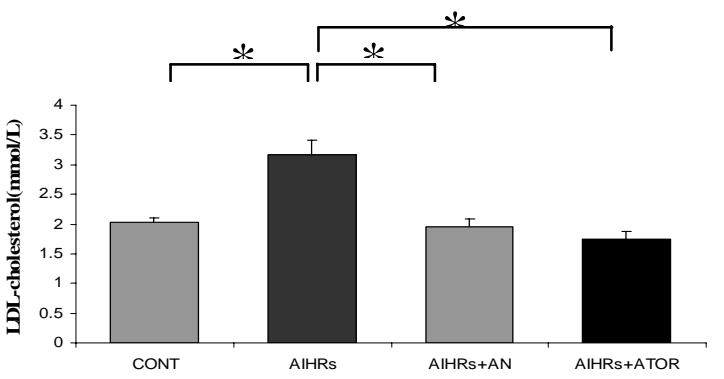

$1 \mathrm{C}$

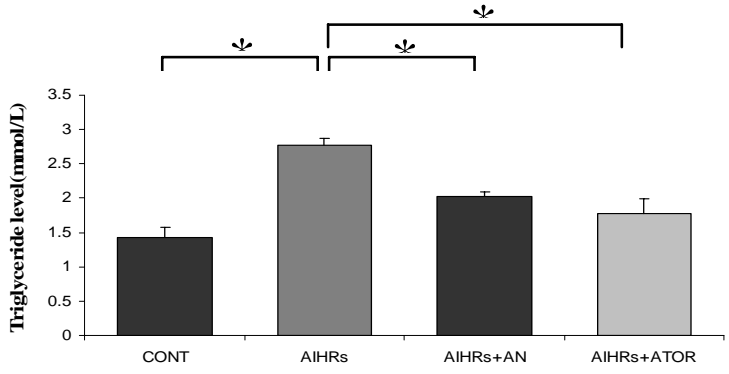

1B

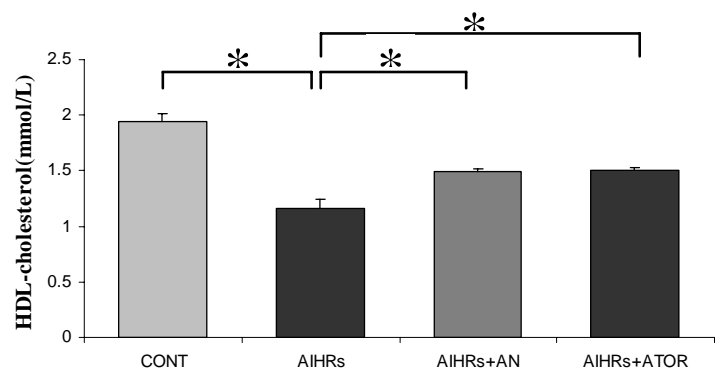

$1 D$

Figure 1. Effects of AN leaf extract on lipid profile in AIHRs for one week. Figures (1A-1D) represent the estimation of TC, TG, LDL, and HDL-C levels, respectively, after i.p administration (30 mg/150 gm b.w.) of AN in AIHRs for one week. All values are presented as mean \pm SEM, $\mathrm{n}=4$ in each case. ${ }^{*} P<0.05$ vs control. ${ }^{* *} P<0.01$ vs AIHRs. ATOR $=$ Atorvastatine, AN $=$ Acacia nilotica leaf extract

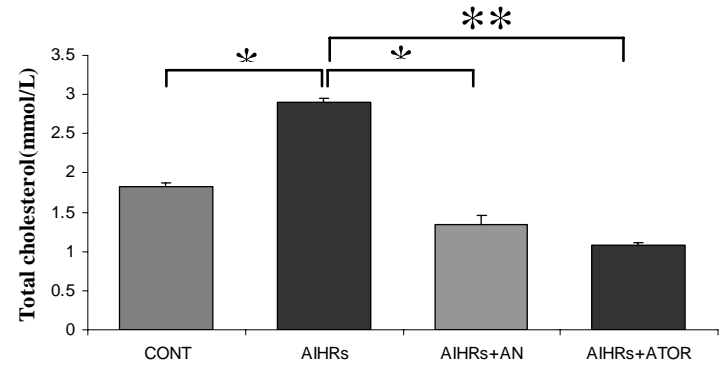

2A

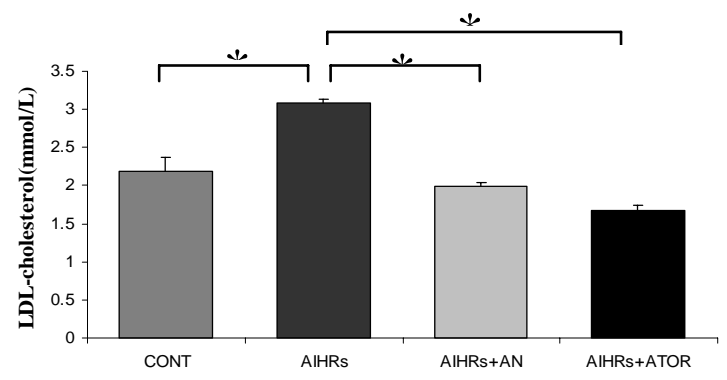

2C

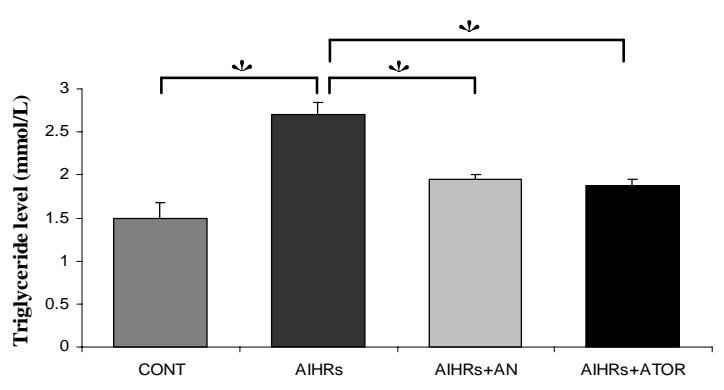

2B

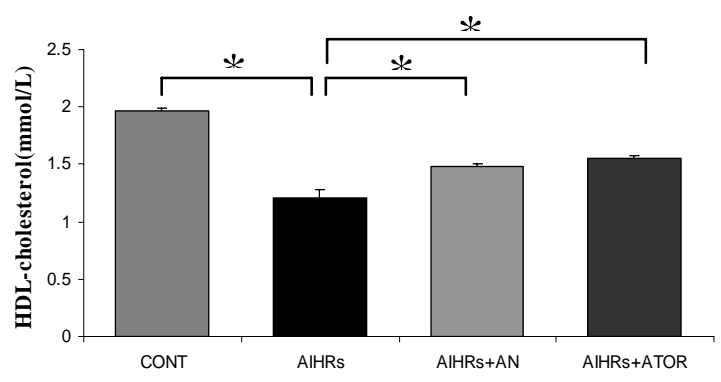

2D

Figure 2. Effects of AN leaf extract on lipid profile in AIHRs for four weeks. Figures (2A-2D) represent the estimation of TC, TG, LDL, and HDL-C levels, respectively, after i.p administration (30 mg/150 gm b.w.) of AN in AIHRs for two weeks. All values are presented as mean \pm SEM, $\mathrm{n}=4$ in each case. ${ }^{*} \mathrm{P}<0.05$ vs control. ${ }^{* *} \mathrm{P}<0.01$ vs AIHRs. ATOR $=$ Atorvastatine, $\mathrm{AN}=$ Acacia nilotica leaf extract 
Effects of Acacia nilotica (AN) leaf extract on left ventricular hypertrophy, heart weight, and cardiac myocytes in AIHRs: AN leaf extract as well as atorvastatine significantly reduced left ventricular hypertrophy (0.004 and 0.0038 for one week vs 0.0033 and 0.0028 for four weeks protocol, respectively) in adrenaline induced hyperlipidemic rats (Figure 3A-3B).

Induction of adrenaline in rats for one week significantly increased heart weight $(0.7 \mathrm{gm})$ in comparison with control rats (0.3gm). Treatment with AN extract and atorvastatine reduced heart weight (0.5 and 0.4 gm, respectively) in AIHRs. For four weeks protocol similar results were obtained where adrenaline increased heart weight $(0.9 \mathrm{gm})$ in comparison to control rats

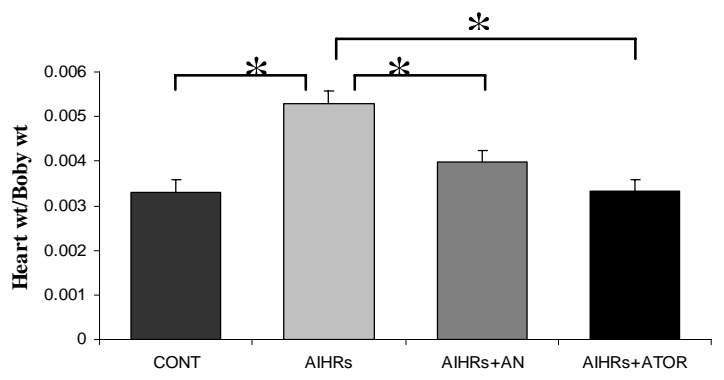

3A

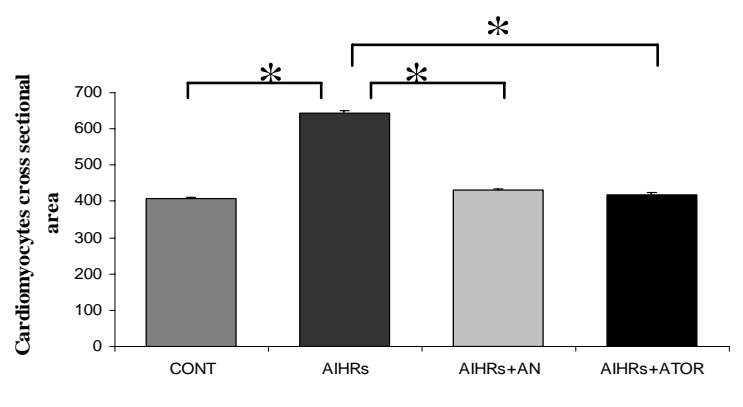

$3 \mathrm{C}$ (0.4gm). Treatment with AN leaves extract and atorvastatine reduced heart weight $(0.6 \mathrm{gm}$ and $0.5 \mathrm{gm}$, respectively).

Figures 3C and 3D demonstrate the effects of AN leaves extract on cardiac myocytes size in AIHRs. Administration of adrenaline in rats for one week as well as four weeks increased cardiac myocytes cross sectional area $\left(639.75\right.$ and $643.75 \mu \mathrm{m}^{2}$ for one and four weeks, respectively) in comparison with control rats (417.5 and $408.75 \mu \mathrm{m}^{2}$, respectively). Treatment with AN leaves extract significantly reduced cardiac myocytes cross sectional area (430 and $431.25 \mu \mathrm{m}^{2}$ for one and four weeks, respectively) compared to atorvastatine (425 and $418.75 \mu \mathrm{m}^{2}$ for one and four weeks, respectively).

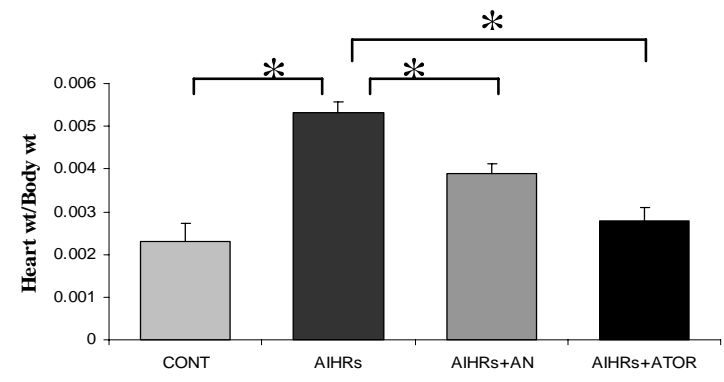

3B

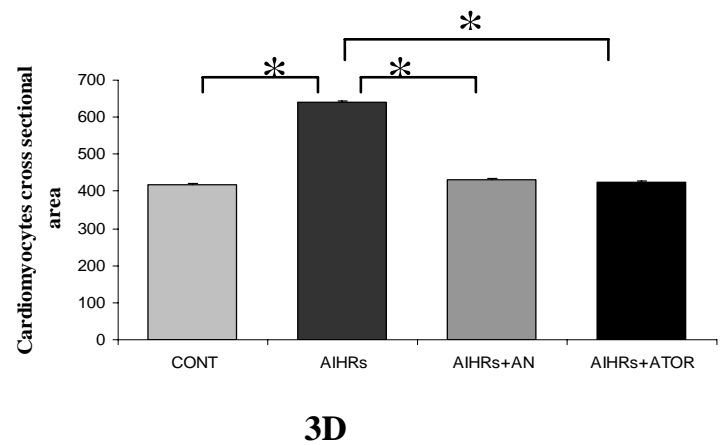

3D

Figure 3. Effects of AN leaf extract on left ventricular hypertrophy and cardiac myocytes in AIHRs for one and four weeks. Figure 3A and 3B represent the effect on left ventricular hypertrophy, whereas 3C and 3D represent the effect on cardiac myocytes in AIHRs for one and 4 weeks, respectively. The data were shown as mean \pm SEM, $n=4$ in each case $* P<0.05$ vs control. ${ }^{* *} P<0.01$ vs AIHRs. ATOR $=$ Atorvastatine, AN $=$ Acacia nilotica leaves extract

Effects of AN leaf extract on kidney, pancreas and liver weight (in gm) on AIHRs for one and four weeks: Figures $4 \mathrm{~A}$ and $4 \mathrm{~B}$ demonstrate the effects of AN leaves extract on kidney, pancreas and liver weight on AIHRs. Infusion of adrenaline in rats for one week significantly increased kidney, pancreas and liver weight $(0.7,0.6$, and 5.2 gm, respectively) in comparison with control (0.4, 0.4 and 3.1 gm, respectively). Treatment with AN leaf extract significantly reduced kidney, pancreas and liver weight (0.6, 0.5 and $4.8 \mathrm{gm}$, respectively) in AIHRs and the result is comparable to atorvastatine $(0.5,0.4$, and $4.2 \mathrm{gm}$, respectively). Similar results were observed for four weeks study (Figure 4B). 


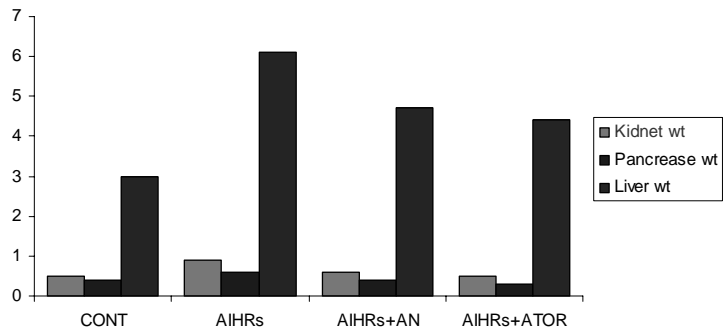

4A

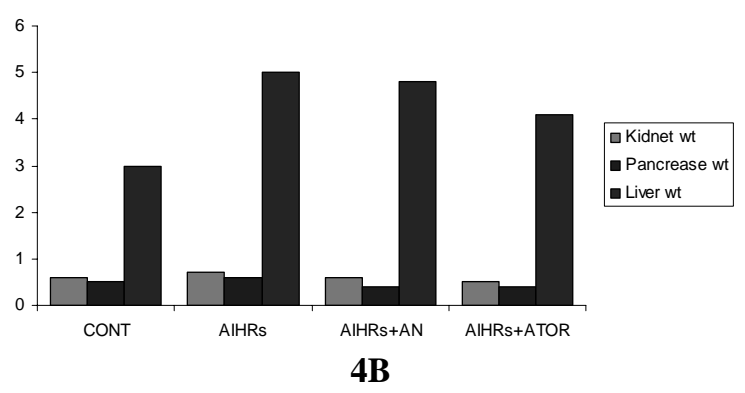

Figure 4. Effects of AN leaf extract on kidney, pancreas and liver weight in gm on adrenaline induced hyperlipidemic rats (AIHRs). 4A and 4B are the effects for one week and four weeks, respectively. ATOR $=$ Atorvastatine

\section{Discussion}

Our study explored that A. nilotica leaf extract reduced high lipid profile and cardiac remodeling in adrenaline induced hyperlipidemic rats as shown in figure 1 to 4 . The present study revealed that $A$. nilotica extract at a dose $30 \mathrm{mg}$ per 150gm body weight of rats has got profound lipid lowering activity and reducing left ventricular hypertrophy, heart weight, cardiac myocytes size when compared with the control rats and AIHRs. Asian herbal medicine reported that most potent constituent of A. nilotica leaf is reserpine which is recommended for the treatment of hyperlipidemia (Jigam et al., 2010) and works by decreasing heart rate and relaxing the blood vessels so that blood can flow more easily through the body. Present study with $A$. nilotica leaf extract also evaluates the hypolipidemic effect in vivo on adrenaline induced hyperlipidemic rats. This relevant experimental study proved that the crude leaf extract has antihyperlipidemic properties and reduce cardiac change. Thus intake of $A$. nilotica leaf extract as medicine might have potential benefit in the treatment of hyperlipidemia and cardiac remodeling.

\section{References}

Aattar, A.A. 2006. Comparative physiological study on the effect of rosemary, tarragon and bay leaves extract on serum lipid profile of quail, Coturnix coturnix. Saudi J. Biol. Sci., 13, 91-98.

Amos, S., Akah, P.A., Odukwe, C.J., Gamaniel, K.S. and Wambede, C. 1999. The pharmacological effects of an aqueous extract from Acacia nilotica seeds. Phytother. Res., 13, 683-685.

Bhopal, R.S. and Rafnsson, S.B. 2007. Mitochondrial efficiency explain the susceptibility to adiposity, metabolic syndrome, diabetes and cardiovascular diseases in South Asian populations. Int. J. Epidem., 31, 239-249.

Ghasi, S., Nwobodo, E. and Ofili, J.O. 2000. Hypocholesterolemic effects of crude extract of leaf of Acacia nilotica in high fat diet fed wistar rats. J. Ethnopharmacol., 69, 2125.

Gilani, A.H., Faizia, S., Siddiqui, B.S., Saleem, R., Aftab, K. and Shaheen, F. 1999. Studies on antihypertensive and antispasmodic activities of methanol extract of Acacia nilotica pods, Phytother. Res., 13, 665-669.

Grabely, S. and Thiericke, R. 1999. Bioactive agents from natural sources: trends in discovery and application. Adv. Biochem. Eng. Biotech., 64, 101-154.

Jigam, A.A., Akanya, H.O., Ogbadoyi, E.O. and Dauda, B.E.N. 2010. In vivo antiplasmodial, analgesic and antiinflammatory effects of the root extracts of Acacia nilotica Del (Leguminosae). Asian J. Exp. Biol. Sci., 1, 315-320.

Lteif, A.A., Mather, K.J. and Clark, C.M. 2003. Diabetes and heart disease an evidence-driven guide to risk factors management in diabetes. Cardiol. Rev. 11, 262-274.

Opie, L.H., Commerford, P.J., Gersh, B.J., Pfeffer, M.A. 2006. Controversies in ventricular remodelling. Lancet, 367, 356.

Richard, D.H., Mary, J.M. 2007. In: Lippincott's llustrated Reviews Pharmacology, $3^{\text {rd }}$ edn. B.I. Publications Pvt. Ltd, Ch. 16, pp. 181-183 \& Ch. 21, pp. 245-246.

Shah, B.H., Safdar, B., Virani, S.S., Nawaz, Z., Saeed, S.A. and Gilani, A.H. 1995. The antiplatelet aggregatory activity of Acacia nilotica is due to blockade of calcium influx through membrane calcium channels. J. Endocrine., 29, 469-470.

Stamler, J., Vaccaro, O., Neaton, J.D. and Wentworth, D. 1993. Diabetes, other risk factors, and 12-yr cardiovascular mortality for men screened in the Multiple Risk Factor Intervention Trial. Diabetes Care, 16, 434-444.

Wilson, P.W. 2001. Diabetes mellitus and coronary heart disease. Endocrinol. Metab. Clin. North. Am., 30, 857-881.

Wong, K.H., Li, G.Q., Li, K.M., Naumovski, V.R. and Chan, K. 2011. Kudzu root: Traditional uses and potential medicinal benefits in diabetes and cardiovascular diseases. $J$. Ethnopharmacol., 134, 584-607. 